\title{
Capacidade inspiratória, limitação ao exercício, e preditores de gravidade e prognóstico, em doença pulmonar obstrutiva crônica*
}

\author{
Inspiratory capacity, exercise limitation, markers of severity, and \\ prognostic factors in chronic obstructive pulmonary disease
}

\author{
Clarice Guimarães de Freitas ${ }^{1}$, Carlos Alberto de Castro Pereira ${ }^{2}$, Carlos Alberto de Assis Viegas ${ }^{3}$
}

\begin{abstract}
Resumo
Objetivo: Correlacionar a capacidade inspiratória $(\mathrm{Cl}), \%$ do previsto, pós-broncodilatador (pós-BD), com outras variáveis indicativas de gravidade e prognóstico, na doença pulmonar obstrutiva crônica (DPOC). Métodos: Oitenta pacientes estáveis com DPOC realizaram manobras de capacidade vital forçada, capacidade vital lenta, e teste de caminhada de 6 min, antes e após salbutamol spray (400 $\mu \mathrm{g})$. Foram divididos em quatro grupos, segundo o volume expiratório forçado no primeiro segundo pós-BD. Diversas variáveis foram testadas, por análise univariada e multivariada, com a distância caminhada pós-BD, \% do previsto. A $\mathrm{Cl}$ pós-BD foi correlacionada com o estadiamento Global Initiative for Chronic Obstructive Lung Disease (GOLD) e o indice Body mass index, airway Obstruction, Dyspnea, and Exercise capacity (BODE). Resultados: Por análise de regressão multivariada, a $\mathrm{Cl}$ pós $\mathrm{BD}, \%$ do previsto, $(\mathrm{p}=0,001)$, o uso de oxigênio a longo prazo ( $p=0,014)$, e o número de medicamentos usados $(\mathrm{p}=0,044)$, mantiveram associação significativa com a distância caminhada, $\%$ do previsto. $\mathrm{A} C \mathrm{Cl} \leq 70 \%$ foi observada em 56\% dos pacientes em estágios GOLD 3 ou 4 comparado a 20\% em estágios GOLD 1 ou $2(\mathrm{p}<0,001)$. A Cl $\leq 70 \%$ foi observada em 60\% dos pacientes com escore BODE 3 ou 4 vs. 33\% com BODE 1 ou 2 ( p = 0,02). Conclusão: A Cl, \% do previsto, pós-BD é o melhor preditor funcional da distância caminhada, associando-se significativamente com o escore GOLD e o índice BODE. Por isso, propomos que a $\mathrm{Cl}$ seja incluída na rotina de avaliação dos portadores de DPOC.
\end{abstract}

Descritores: Doença pulmonar obstrutiva crônica; Caminhada; Testes de função pulmonar; Capacidade inspiratória.

\begin{abstract}
Objective: To correlate the postbronchodilator (post-BD) inspiratory capacity (1C), \% of predicted, with other markers of severity and prognostic factors in chronic obstructive pulmonary disease (COPD). Methods: Eighty stable patients with COPD performed forced vital capacity and slow vital capacity maneuvers, as well as the 6-min walk test, prior to and after receiving albuterol spray (400 $\mu \mathrm{g})$. Patients were divided into four groups, based on post-BD forced expiratory volume in one second. Several variables were tested to establish correlations with the post-BD distance walked, using univariate and multivariate analysis. Post-BD 1C was found to correlated with Global lnitiative for Chronic Obstructive Lung Disease (GOLD) staging and with the Body mass index, airway Obstruction, Dyspnea, and Exercise capacity (BODE) index. Results: Multivariate regression analysis revealed that the distance walked, \% predicted, correlated significantly with the $1 \mathrm{C}$ post-BD, $\%$ predicted $(p=0.001)$, long-term oxygen use $(p=0.014)$, and number of medications used in the treatment $(p=0.044) .1 C \leq 70 \%$ was observed in 56\% patients in GOLD stages 3 or 4 vs. 20\% in GOLD 1 or 2 ( $p<0.001)$. 1C $\leq 70 \%$ was observed in (60\%) patients with BODE score 3 or 4 vs. $(33 \%)$ BODE score 1 or $2(p=0.02)$. Conclusion: Post-BD 1C\% predicted is the best functional predictor of distance walked and is significantly associated with GOLD staging and BODE index. Therefore, We propose that the inspiratory capacity should be added to the routine evaluation of the COPD patients.
\end{abstract}

Keywords: Pulmonary disease, chronic obstructive; Walking; Respiratory function tests; Inspiratory capacity.

\footnotetext{
* Trabalho realizado na Universidade de Brasília e Hospital de Base do Distrito Federal Brasília (DF) Brasil.

1. Doutora em Ciências da Saúde na area de Pneumologia pela Universidade de Brasília - UNB - Brasilia (DF) Brasil.

2. Doutor em Pneumologia pela Escola Paulista de Medicina da Universidade Federal de Sao Paulo - UNIFESP-EPM - São Paulo (SP) Brasil.

3. Professor Associado da Faculdade de Medicina da Universidade de Brasília - UNB - Brasilia (DF) Brasil.

Endereço para correspondência: Clarice Guimarães de Freitas. Hospital de Base do Distrito Federal, SQN 310, Bloco L, apto 107, Asa Norte, CEP 70756-120, Brasilia, DF, Brasil.

Tel 5561 3325-4815. Email: claricegfs@terra.com.br

Recebido para publicação em 18/9/06. Aprovado, após revisão, em 23/10/06.
} 


\section{Introdução}

A doença pulmonar obstrutiva crônica (DPOC) é uma enfermidade comum, geralmente progressiva e debilitante, caracterizada por limitação do fluxo aéreo. Esta doença não é totalmente reversível e está associada a uma resposta inflamatória do pulmão a partículas ou gases nocivos. ${ }^{(1)}$

0 estadiamento da DPOC é importante para estabelecer o prognóstico e hierarquizar o tratamento. Um sistema de estadiamento ideal deve ter forte correlação com a mortalidade, morbidade e estado de saúde. Tradicionalmente, a gravidade da DPOC é definida pelo grau de obstrução, avaliada pelo volume expiratório forçado no primeiro segundo $\left(\mathrm{VEF}_{1}\right)$ após uso de broncodilatador (pós$\mathrm{BD})$. Os pontos de corte sugeridos para as medidas do $\mathrm{VEF}_{1}$ para o estadiamento são variáveis, havendo diferenças significativas entre importantes sociedades de pneumologia. ${ }^{(2-4)}$ Entretanto, o $\mathrm{VEF}_{1}$ não se correlaciona bem com a dispnéia, que é o sintoma mais importante do paciente portador de DPOC. ${ }^{(5)}$ $0 \mathrm{VEF}_{1}$ também não é bom marcador de sobrevida, freqüência de internações e capacidade funcional, principalmente em pacientes mais graves. Além disso, o paciente portador de DPOC tem manifestações sistêmicas que não são representadas pelo $\mathrm{VEF}_{1}$.

Estudos recentes sugerem que outros parâmetros além do $\mathrm{VEF}_{1}$, como o índice de massa corpórea (IMC), grau de dispnéia, distância caminhada avaliada pelo teste de caminhada de 6 min (TC6), qualidade de vida relacionada à saúde, pico de consumo de oxigênio $\left(\mathrm{VO}_{2}\right)$, a relação capacidade inspiratória/capacidade pulmonar total (Cl/CPT - indice inspiratório), o índice Body mass index, airway Obstruction, Dyspnea, and Exercise capacity (BODE), ${ }^{(6)}$ e o uso de oxigênio a longo prazo, independentemente, são importantes fatores preditores de prognóstico em DPOC, e alguns são, na verdade, melhores marcadores que o $\mathrm{VEF}_{1} \cdot{ }^{(7-9)}$

Diversos fatores respondem pela limitação de exercício em pacientes com DPOC; porém, têm papel de destaque a limitação ventilatória e a muscular periférica. ${ }^{(10,11)} \mathrm{Na}$ DPOC, a perda da capacidade elástica pulmonar e a limitação expiratória progressiva promovem aprisionamento aéreo, com aumento da capacidade residual funcional e diminuição da $\mathrm{Cl}$. A hiperinsuflação estática e seu aumento durante o exercício (hiperinsuflação dinâmica) se associam à limitação ao exercício em portadores da DPOC. ${ }^{(11,12)} \mathrm{A}$ redução da hiperinsuflação, expressa pelo aumento da $\mathrm{Cl}$ pós-BD, se correlaciona com melhor tolerância ao exercício e redução da dispnéia. ${ }^{(13)}$ Existe, ainda, correlação entre maior mortalidade e diminuição da relação $\mathrm{Cl} / \mathrm{CPT} .{ }^{(14)}$

Além da hiperinsuflação dinâmica e fraqueza muscular esquelética, outros fatores contribuem para a limitação ao exercício, como anormalidades da troca gasosa e hipertensão pulmonar. Além disso, pacientes com DPOC freqüentemente apresentam doenças relacionadas ao tabagismo, tais como hipertensão e doença cardíaca, que também contribuem para a limitação ao esforço. 0 TC6 reflete todos esses fatores, incluindo os efeitos sistêmicos associados à doença e à limitação ventilatória. ${ }^{(15)}$

Entre os diversos parâmetros funcionais respiratórios, seria interessante selecionar aquele que mais se correlaciona com a capacidade de exercício, para inclusão em sistemas de estadiamento, já que a contribuição da parte ventilatória pode variar, independentemente do grau de limitação periférica.

0 objetivo do estudo foi avaliar a correlação da $\mathrm{Cl}$ (\% previsto pós-BD) e outros parâmetros de limitação ventilatória, como a capacidade vital forçada (CVF), $\mathrm{VEF}_{1}$ (\% previsto pós-BD), $\mathrm{VEF}_{1} / \mathrm{CVF}$, dispnéia pós-TC6, número de exacerbações, uso de oxigênio, e número de drogas utilizadas no tratamento, com variáveis indicadoras de prognóstico e de gravidade da DPOC, como a distância caminhada em 6 min (\% previsto pós-BD), o índice BODE e o sistema de estadiamento proposto pelo Global Initiative for Chronic Obstructive Lung Disease (GOLD).

\section{Pacientes e métodos}

Trata-se de estudo transversal, descritivo, no qual foram avaliados 80 pacientes estáveis, portadores de DPOC. Os critérios de inclusão foram: idade acima de 35 anos, história de tabagismo de mais de 20 maços-ano, e resultado da avaliação funcional $\mathrm{VEF}_{1} / \mathrm{CVF}$ (\% do previsto) pós-BD menor que 70\%. Os pacientes foram selecionados em dois Serviços de Pneumologia de Brasília e foram acompanhados durante pelo menos dois meses pelo primeiro autor do estudo, apresentando boa adesão ao tratamento, que foi instituído segundo os sintomas e a gravidade, de acordo com as diretrizes propostas pelo GOLD. (1) Os critérios de exclusão foram: doença instável; uso de altas doses de corticosteróide oral (40 mg de prednisona ou mais); uso de antibióticos 
durante as últimas quatro semanas antes do estudo; ou co-morbidades incontroladas, como miopatias, doença vascular periférica, doenças reumáticas ou ortopédicas, que impediam a locomoção, bem como doença isquêmica cardíaca ou arritmia.

Os pacientes foram divididos de acordo com o grau de obstrução, segundo o VEF pós-BD, em quatro grupos: leve, moderado, grave, e muito grave, como proposto pelo consenso GOLD. (1) Também foram divididos em quatro grupos segundo o índice BODE, que define o risco de morte. ${ }^{(2)}$ Eles foram submetidos à espirometria tradicional, incluindo a medida da capacidade vital lenta e da $\mathrm{Cl}$. Após a espirometria, o TC6 foi realizado, de acordo com as normas propostas pela American Thoracic Society, com adição de medida da saturação periférica de oxigênio ( $\mathrm{SpO}_{2}$, pelo oxímetro de pulso) antes, durante e depois do teste. ${ }^{(16)}$ Todos os testes foram repetidos, 15 min após inalação de salbutamol spray (400 $\mu \mathrm{g})$. Ao fim do TC6, a dispnéia era avaliada pela escala de Borg. 0 ritmo da caminhada era determinado pelos pacientes, que eram acompanhados e incentivados com estímulos verbais, padronizados pelo pneumologista. Os pacientes que faziam uso de oxigênio domiciliar caminhavam em uso de oxigênio.

Os valores previstos para a manobra de CVF e para a $\mathrm{Cl}$ foram baseados nos valores sugeridos para a população brasileira. ${ }^{(17,18)}$ Os valores preditos para a distância caminhada foram os já estabelecidos em literatura. ${ }^{(19)}$
Diversas variáveis foram testadas, por análise univariada (teste de Spearman e Pearson) e multivariada, com a distância caminhada pós-BD, expressa como percentual do previsto. A normalidade da distribuição das variáveis foi testada pelo teste de Kolmogorov-Smirnov. Àquelas que tiveram distribuição normal foi aplicado teste paramétrico e àquelas de distribuição não normal, teste não paramétrico. Foi considerado de significância estatística o valor de $p \leq 0,05$. Os cálculos foram feitos pelo programa SPSS 12.0 (SPSS, Chicago, IL, EUA).

Os pacientes concordaram em participar do estudo, assinando o termo de consentimento esclarecido, e o protocolo foi aprovado pelo comitê de ética da Faculdade de Ciências da Saúde, Universidade de Brasília.

\section{Resultados}

Os dados gerais dos 80 pacientes portadores de DPOC são mostrados na Tabela 1.

Foram avaliados 57 homens e 23 mulheres. Deste total, 5 pacientes (6\%) tinham obstrução leve, 29 (36\%) moderada, 34 (43\%) grave, e 12 (15\%) muito grave, de acordo com a classificação proposta pelo GOLD. Quanto ao índice BODE, 32 pacientes (40\%) situavam-se no quartil 1, 25 (31\%) no quartil $2,17(21 \%)$ no quartil 3 e $6(8 \%)$ no quartil 4 . Havia 15 pacientes (19\%) com IMC $\leq 21 \mathrm{~kg} / \mathrm{m}^{2}$. 0 IMC se correlacionou negativamente com o escore GOLD

Tabela 1 - Dados gerais em 80 pacientes com doença pulmonar obstrutiva crônica.

\begin{tabular}{lcc}
\hline \multicolumn{1}{c}{ Variáveis } & Média $\pm \mathrm{dp}$ ou $\%$ & Valores mínimos/máximos \\
\hline ldade (anos) & $70 \pm 8$ & $52 / 86$ \\
Sexo masculino (\%) & 71 & $15 / 40$ \\
IMC $\left(\mathrm{kg} / \mathrm{m}^{2}\right.$ ) & $25 \pm 4$ & $1 / 5$ \\
Uso de oxigênio (\%) & 9 & $20 / 145$ \\
Numero de exacerbações/ano & 2 & $43 / 136$ \\
Tabagismo (maços/ano) & $51 \pm 24$ & $15 / 101$ \\
CVF pós-BD (\% previsto) & $80 \pm 16$ & $28 / 69$ \\
VEF ${ }_{1}$ pós-BD (\% previsto) & $49 \pm 18$ & $37 / 157$ \\
VEF $/$ CVF\% (encontrado) & $49 \pm 11$ & $22-137$ \\
Cl pós-BD (\% previsto) & $78 \pm 22$ & $88 / 606$ \\
Distância caminhada em 6 min pósBD (\% previsto) & $88 \pm 21$ & $74 / 95$ \\
Distância caminhada em 6 min pósBD (m) & $397 \pm 99$ & $88 \pm 5$ \\
Sp0 6 min pós-BD (\%)
\end{tabular}

${ }^{a} \mathrm{dp}$ = desvio padrão; IMC: indice de massa corpórea; VEF, pós-BD: Volume expiratório forçado no primeiro segundo após uso de broncodilatador; CVF pós-BD: Capacidade vital forçada após uso de broncodilatador; $\mathrm{VEF}_{1} / \mathrm{CVF} \%$ : Volume expiratório forçado no primeiro segundo/Capacidade vital forçada após uso de broncodilatador, percentual, encontrado; $\mathrm{Cl}$ pós-BD: Capacidade inspiratória após uso de broncodilatador, $\mathrm{SpO}_{2} 6$ min pós BD: Saturação de oxigenio a oximetria de pulso após uso de broncodilatador, após TC6. 
de maneira significativa, porém fraca $(r=-0,26$ $\mathrm{p}=0,017$ ), e com o índice BODE também de maneira inversa, mas também pobre $(r=-0,27$, $p=0,015)$.

A distância caminhada em 6 min, \% do previsto, não foi diferente entre sexos e não se correlacionou de maneira significativa com a idade, com a estatura, e nem com o IMC. Nos pacientes com $\mathrm{IMC}<21 \mathrm{~kg} / \mathrm{m}^{2}$, a distância caminhada em 6 min foi de $81 \pm 16$, \% do previsto, em comparação a $89 \pm 22 \%$ dos demais pacientes $(t=1,32$, $\mathrm{p}=0,19)$.

Comorbidades tais como hipertensão arterial sistêmica, doença coronariana, arritmia, diabetes, e hipotireoidismo, estavam presentes em 42 pacientes. A distância caminhada em 6 min, \% do previsto, neste grupo, de pacientes com comorbidades, foi de $84 \pm 20 \%$, em comparação a $91 \pm 22 \%$ nos demais $(p=0,11)$. Os sete pacientes em uso de oxigênio domiciliar a longo prazo percorreram menor distância no TC6, expresso em \% do previsto, quando comparados aos pacientes sem uso de oxigênio ( $47 \pm 20 \%$ vs. $92 \pm 17 \%, t=6,70, p=0,0001)$. Houve correlação inversa entre a distância caminhada em 6 min, $\%$ do previsto, e o número de exacerbações anuais $\left(r_{s}=-0,29, p=0,009\right)$, bem como entre a distância caminhada em $6 \mathrm{~min}$, \% do previsto, e o número de drogas utilizadas no tratamento $\left(r_{\mathrm{s}}=-0,46\right.$, $p=0,0001)$.

Dentre as variáveis funcionais, obtidas após administração de broncodilatador, as correlações com a distância caminhada em 6 min, \% do previsto, observadas foram: CVF \% do previsto $(r=0,42, p<0,00), V_{E F} \%$ do previsto $(r=0,44$, $p<0,001), V E F_{1} / C V F \%$ do previsto $(r=0,47$, $p<0,001)$, número de medicamentos usados no tratamento $(r=-0,49, p<0,001)$, GOLD $(r=-0,52$, $\mathrm{p}<0,001)$, índice de BODE $(-0,60, \mathrm{p}<0,001)$, $\mathrm{Cl}$, $\%$ do previsto, $(r=0,61, p<0,001)$, Borg pós 6 min $(r=-0,41, p<0,001), \mathrm{SpO}_{2}(r=0,31, p=0,005)$ ao término do teste. Por análise de regressão múltipla três variáveis mantiveram associação significativa com a distância caminhada em 6 min, \% do previsto: $\mathrm{Cl}$ pós-BD \% do previsto $(\mathrm{p}<0,001)$, uso a longo prazo de oxigênio $(p=0,001)$ e número de medicamentos usados no tratamento $(p=0,034)$. Por regressão multivariada anterógrada, estas três variáveis explicaram 59\% da variação na distância caminhada em 6 min, a $\mathrm{Cl}$ sendo responsável por $38 \%$ desta variação. Adicionando-se o uso de oxigênio, o coeficiente de explicação se elevou para 55\%; e o uso de medicações, para 59\%. Retirandose os sete pacientes que faziam uso de oxigênio, e repetindo-se a análise multivariada anterógrada, permaneceram significativas a $\mathrm{Cl}, \%$ do previsto, a dispnéia, medida pela escala de Borg após a caminhada, e o número de medicamentos utilizados no tratamento. Novamente, a $\mathrm{Cl}, \%$ do previsto, respondeu por 38\% desta variação; em segundo lugar, a dispnéia, elevando o $r^{2}$ para 51\%. A correlação entre a $\mathrm{Cl}$ e a distância caminhada em 6 min, $\%$ do previsto, é mostrada na Figura 1.

Os pacientes em uso de corticosteróide oral percorreram menor distância caminhada em 6 min, $\%$ do previsto, em relação àqueles que não usavam corticosteróides (62 $\pm 24 \%$ vs. $92 \pm 18 \%, \mathrm{t}=4,73$, $\mathrm{p}=0,000)$ e apresentaram menor $\mathrm{VEF}_{1}$ pós $\%$ do previsto $(32 \pm 16 \%$ vs. $51 \pm 17 \%, \mathrm{t}=3,44$, $\mathrm{p}=0,001)$. Por análise multivariada, o uso de corticosteróide oral permaneceu significativo em relação à distância caminhada, quando o $\mathrm{VEF}_{1}$ e o uso de oxigênio e demais fatores relevantes foram incluídos $(\mathrm{p}=0,016)$.

Trinta e três pacientes tinham $\mathrm{Cl}$ pós- $\mathrm{BD} \leq$ $70 \%$ do previsto. $\mathrm{Cl} \leq 70 \%$ foi observada em 26/46 pacientes (56\%) em estágios GOLD 3 ou 4, em comparação a $7 / 34$ pacientes (20\%) em estágios GOLD 1 ou $2\left(\chi^{2}=10,41, p=0,001\right)$, Figura 2 . $\mathrm{Cl} \leq 70 \%$ foi observada em $14 / 23$ pacientes (60\%) com escore BODE 3 ou 4, em comparação a 19/57 pacientes BODE 1 ou $2(33 \%)\left(\chi^{2}=5,127, p=0,02\right)$, Figura 3.

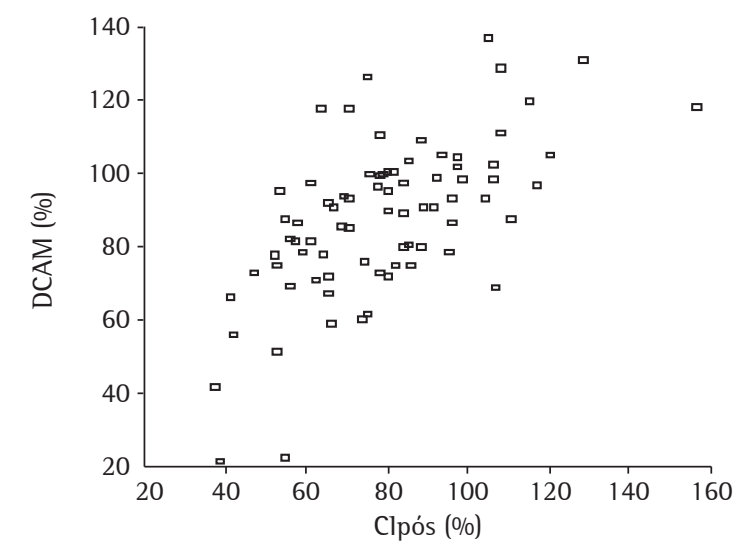

Figura 1 - Correlação entre a distância caminhada em 6 min (DCAM (\%)) e a capacidade inspiratória após uso de broncodilatador (Clpós (\%)). 


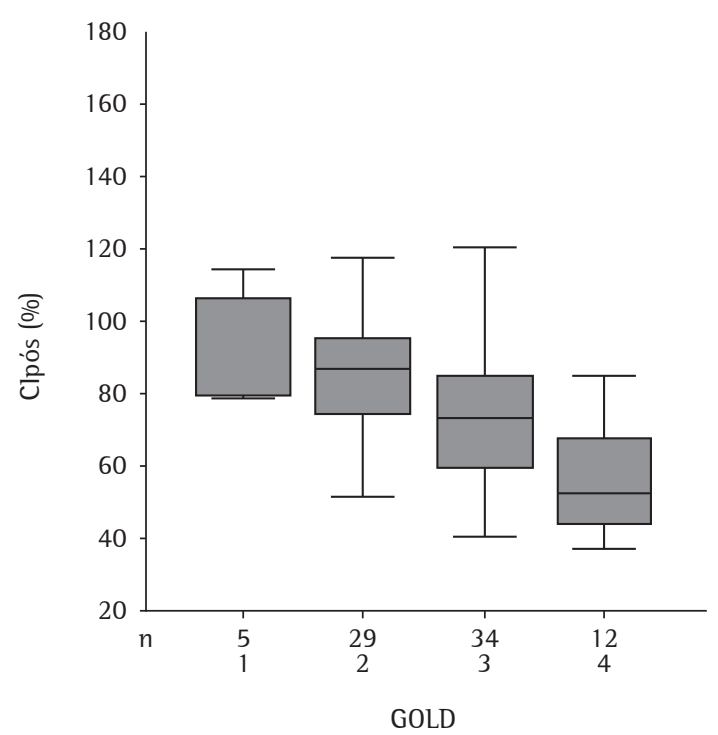

Figura 2 - Relação entre a capacidade inspiratória após uso de broncodilatador (Clpós (\%)) e o estadiamento de gravidade Global Initiative for Chronic Obstructive Lung Disease (GOLD).

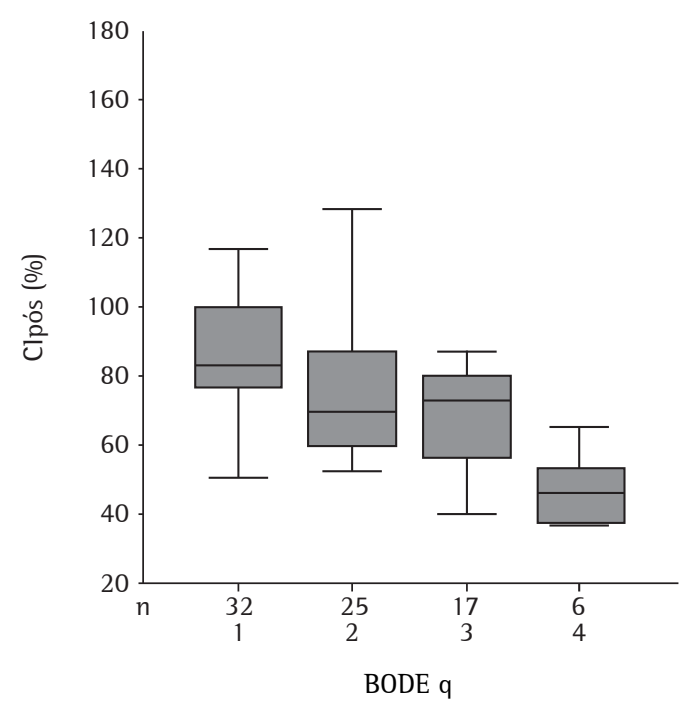

Figura 3 - Relação entre a capacidade inspiratória após uso de broncodilatador (Clpós (\%)) e o índice Body mass index, airway Obstruction, Dyspnea, and Exercise capacity (BODE), separado por quartis.

\section{Discussão}

Os resultados apresentados sugerem que a $\mathrm{Cl}$, $\%$ do previsto, pós-BD é um bom marcador de gravidade e prognóstico na DPOC, já que houve correlação forte e significativa entre $\mathrm{Cl}, \%$ do previsto, e distância caminhada em 6 min, \% do previsto, e associações significativas entre a $\mathrm{Cl}$ e os estágios de gravidade propostos pelo GOLD, e o escore de prognóstico expresso pelo índice BODE.

Os estágios de gravidade pelo GOLD levam em conta o grau de obstrução baseado no VEF $\%$ do previsto pós-BD. (1) Os estágios GOLD 3 e 4 , ou o $\mathrm{VEF}_{1} \%$ do previsto $<50 \%$, correlacionaram-se com $\mathrm{Cl}, \%$ do previsto, $<70 \%$.

0 índice BODE contém um componente que quantifica o distúrbio ventilatório pelo $\mathrm{VEF}_{1}$, outro que capta a percepção de dispnéia, além de outros componentes independentes, que são a distância caminhada em 6 min e o IMC, os quais refletem as conseqüências sistêmicas da DPOC. ${ }^{(6)} 0$ escore de pontos baseado nestas variáveis prediz melhor o prognóstico da doença, em comparação ao $\mathrm{VEF}_{1}$ isolado. ${ }^{(6)}$ A diminuição do IMC é um bom marcador de comprometimento sistêmico da DPOC e se associa a maior mortalidade ${ }^{(6)}$; porém, não encontramos forte correlação entre o IMC e a distância caminhada em $6 \mathrm{~min}$, \% do previsto. Talvez o número reduzido de pacientes com IMC menor que $21 \mathrm{~kg} / \mathrm{m}^{2}$, apenas 15 , tenha influenciado este resultado.

0 TC6 vem sendo cada vez mais utilizado para complementar a avaliação funcional dos pacientes com DPOC, já que reflete as manifestações sistêmicas da doença e a limitação ventilatória. A variação da distância caminhada com o tempo se correlaciona com mudanças na espirometria e com a sobrevida dos pacientes portadores de DPOC. Um estudo longitudinal recente ${ }^{(20)}$ descreveu pela primeira vez o valor prognóstico da distância caminhada em 6 min em pacientes com DPOC grave. Independente das comorbidades associadas, a distância caminhada em 6 min foi melhor preditor de mortalidade em comparação ao $\mathrm{VEF}_{1}$ e IMC. Na doença avançada, o declínio da distância caminhada em 6 min é independente da queda do $\operatorname{VEF}_{1}{ }^{(14,20)}$ Uma limitação do presente estudo foi não ter correlacionado $\mathrm{a} \mathrm{Cl}$, $\%$ do previsto, e a distância caminhada em 6 min, $\%$ do previsto, com a mortalidade, o que reforçaria o valor da $\mathrm{Cl}$ como marcador de gravidade da doença. Cinco $(8 \%)$ pacientes foram a óbito durante o período de coleta de dados.

Por análise univariada, encontramos correlação significativa inversa entre a distância caminhada em 6 min, \% do previsto, e o número de exacerbações por ano, uso a longo prazo de oxigênio, número de drogas utilizadas para tratamento da DPOC, 
especialmente o uso de corticosteróide sistêmico, e escore de dispnéia medida pela escala de Borg ao término do teste, bem como correlação direta da distância caminhada em 6 min, \% do previsto, com a CVF \% do previsto, $\mathrm{VEF}_{1} \%$ do previsto, $\mathrm{VEF}_{1} / \mathrm{CVF}$ $\%$ do previsto, $\mathrm{Cl}, \%$ do previsto, e $\mathrm{SpO}_{2}$, medida ao término do teste (todos pós-BD).

A distância caminhada pode ser influenciada por altura, peso, idade, sexo, motivação, realização prévia do exame, drogas utilizadas antes do teste, comorbidades, força muscular periférica, e uso de oxigênio suplementar. ${ }^{(16)}$ Pela influência das variáveis antropométricas sobre a distância caminhada em $6 \mathrm{~min}$, adotamos os valores previstos sugeridos na literatura, para a população norte-americana. ${ }^{(19)}$ Os valores de referência para o teste de caminhada não estão disponíveis para a população brasileira. Alguns estudos avaliaram a influência de diversas variáveis sobre a distância caminhada, em DPOC; dependendo do estudo, algumas variáveis foram medidas, e outras ignoradas. Em uma análise multivariada dos fatores que poderiam influenciar a distância caminhada em 6 min, em um estudo envolvendo 83 pacientes com DPOC, ${ }^{(21)}$ observouse que quatro fatores explicavam $78 \%$ da variância total dos dados: o padrão de freqüência cardíaca, a capacidade de endurance, a capacidade de transporte de oxigênio, e a dispnéia. $\mathrm{A} \mathrm{Cl}$ não foi incluída na análise dos dados. A força muscular, incluindo a força dos músculos respiratórios e das extremidades superiores, e a medida da dispersão do monóxido de carbono, influenciam de maneira significativa o desempenho no teste de caminhada em DPOC ${ }^{(22-24)}$; porém, não foram medidas no presente estudo.

Neste estudo, encontramos correlação inversa significativa entre o número de exacerbações e a distância caminhada em $6 \mathrm{~min}$, \% do previsto. $\mathrm{Na}$ DPOC, há redução significativa da força muscular esquelética em pacientes internados durante exacerbação, em comparação ao estado estável, com recuperação apenas parcial, após três meses da internação, o que pode ser atribuído a diversos fatores. ${ }^{(25)} 0$ número de exacerbações é fator de risco de mortalidade, assim como o uso a longo prazo de corticosteróides. ${ }^{(26)}$ Encontramos um valor significativamente menor da distância caminhada em 6 min, $\%$ do previsto, no grupo de pacientes em uso de corticosteróides sistêmicos. Também encontramos distância caminhada em 6 min, \% do previsto, significativamente menor no grupo em uso de oxigênio a longo prazo, em comparação ao dos não usuários de oxigênio. Essa diferença foi subestimada, já que estes pacientes fizeram o teste em uso de oxigênio, o que sabidamente aumenta a distância caminhada em 6 min.

Alguns autores ${ }^{(14)}$ mostraram que a hiperinsuflação, estimada pela razão $\mathrm{Cl} / \mathrm{CPT}$, denominada de índice inspiratório, é um preditor de sobrevida a longo prazo em DPOC, independente do $\mathrm{VEF}_{1}$. Este achado foi confirmado no estudo de coorte de longo prazo do National Emphysema Treatment Trial Research Group, ${ }^{(7)}$ que determinou os fatores de risco de maior mortalidade em pacientes com enfisema grave. Foram identificados como marcadores de pior prognóstico e preditores de maior mortalidade: idade avançada, diminuição do IMC, uso de oxigênio, diminuição da capacidade para o exercício, definida pela carga máxima atingida em cicloergômetro, enfisema predominante em lobos inferiores, além da diminuição da relação $\mathrm{Cl} / \mathrm{CPT}$ e índice BODE modificado. A medida da relação $\mathrm{Cl} / \mathrm{CPT}$ pressupõe a medida da CPT por pletismografia, disponível em poucos centros. $\mathrm{A} \mathrm{Cl} \%$ do previsto, é um teste não invasivo e pode ser medida facilmente pela manobra de capacidade vital lenta, mas critérios rigorosos para sua reprodutibilidade devem ser observados. ${ }^{(27)}$ A não disponibilidade dos valores previstos para a $\mathrm{Cl}$ é uma limitação em diversos países, o que não ocorre em nosso meio. ${ }^{(18)}$ A maior parte dos estudos estima a $\mathrm{Cl}$ não pela espirometria simples, mas pela análise de volumes pulmonares..$^{(7,14)}$

Nos últimos anos, o papel da hiperinsuflação na geração da dispnéia e limitação ao exercício em DPOC foi claramente estabelecido. ${ }^{(28)} \mathrm{A}$ medida da $\mathrm{Cl}$ reflete o grau de hiperinsuflação na DPOC, mas também é influenciada pela força dos músculos inspiratórios, e pela extensão da carga mecânica imposta a estes músculos. $\mathrm{A} \mathrm{Cl}$ também fornece informações a respeito da posição do volume corrente na curva de pressão-volume do sistema respiratório: quanto menor a $\mathrm{Cl}$, mais próximo o volume corrente está da CPT, região em que a relação pressão-volume se torna alinear, aumentando o trabalho elástico da respiração. No exercício, com o aumento da freqüência respiratória, ocorre aumento do ar aprisionado na DPOC, e aumento do volume corrente, com maior demanda elástica para a inspiração. A hiperinsuflação ao exercício foi demonstrada em DPOC, com o teste de caminhada. Não é surpreen- 
dente, portanto, no presente estudo, a observação de que o maior determinante da distância caminhada em 6 min tenha sido a $\mathrm{Cl}$.

Em um estudo, ${ }^{(5)}$ a dispnéia foi um melhor preditor de sobrevida em cinco anos do que o grau de obstrução ao fluxo aéreo, em pacientes com DPOC, e deve ser incluída como variável para a avaliação de mortalidade nestes pacientes. Diversos estudos prévios demonstraram que a dispnéia tem importante papel na determinação da distância caminhada em DPOC. (24,29) $^{-2}$

Outros autores ${ }^{(12)}$ mediram a $\mathrm{Cl}$ em repouso e ao final do teste de caminhada em pacientes com DPOC, e demonstraram, à semelhança do mostrado previamente em testes em cicloergômetro, que a queda da $\mathrm{Cl}$ durante o esforço, que reflete a hiperinsuflação, se correlaciona com a dispnéia, medida pela escala de Borg. Os dados foram obtidos antes do uso de $\mathrm{BD}$, sendo a correlação entre a $\mathrm{Cl}$ e a distância caminhada em 6 min significativa, porém menor $(r=0,41)$ do que a observada no presente estudo $(r=0,61)$. Consideramos sempre todas as variáveis funcionais após o uso do broncodilatador, já que os escores de gravidade sugerem que o paciente deve ser classificado após o alívio do broncoespasmo. Isto pode, também, ter resultado em melhor aprendizado para a realização do teste da distância caminhada, e em melhor correlação com a $\mathrm{Cl}$.

Um artigo recente de revisão questiona a validade da avaliação de rotina da hiperinsuflação dinâmica pela medida da $\mathrm{Cl}$ durante o exercício, através de ergoespirometria. ${ }^{(30)} 0$ autor ressalta o valor do teste de exercício cardiopulmonar na DPOC e, principalmente, a avaliação da dispnéia referida pelo paciente após esforço, que seria uma conseqüência da hiperinsuflação dinâmica, de aferição mais simples. Em nosso estudo, a dispnéia se correlacionou de maneira significativa com a distância caminhada, $\%$ do previsto, além da $\mathrm{Cl}, \%$ do previsto, o que demonstra que a dispnéia tem causa multifatorial em DPOC, não sendo explicada completamente pela hiperinsuflação durante o exercício, embora este seja o mecanismo mais importante.

Em conclusão, a $\mathrm{Cl}, \%$ do previsto, pós-BD é um bom preditor de distância caminhada em 6 min, e tem forte associação com outras variáveis de gravidade e prognóstico em DPOC, como o estadiamento GOLD e o índice BODE. Sugerimos que a $\mathrm{Cl}$ $\%$ do previsto pós-BD deva ser incluída na avaliação funcional de rotina dos pacientes com DPOC. Um ponto de corte de $70 \%$ pode ser usado para classificar os pacientes.

\section{Referências}

1. Pauwels RA, Buist AS, Calverley PM, Jenkins CR, Hurd SS; GOLD Scientific Committee. Global strategy for the diagnosis, management, and prevention of chronic obstructive pulmonary disease. NHLBI/WHO Global Initiative for Chronic Obstructive lung Disease (GOLD) Workshop summary. Am J Respir Crit Care Med. 2001;163(5):1256.

2. Celli BR. The importance of spirometry in COPD and asthma: Effect on approach to management. Chest. 2000;117(2 Suppl):S15-S9.

3. Standards for the diagnosis and care of patients with chronic obstructive pulmonary disease. American Thoracic Society. Am J Respir Crit Care Med. 1995;152 (5 Pt 2):S77-S121

4. BTS guidelines for the management of chronic obstructive pulmonary disease. The COPD Guidelines Group of the Standards of Care Committee of the BTS. Thorax. 1997;52(suppl 5):S1-S28.

5. Nishimura K, lzuni T, Tsukino M, Oga T. Dyspnea is a better predictor of survival than airway obstruction in patients with COPD. Chest. 2002;121(5):1434-40

6. Celli BR, Cote CG, Marin JM, Casanova C, Montes de Oca M, Mendez RA, et al. The body-mass index, airflow obstruction, dyspnea, and exercise capacity index in chronic obstructive pulmonary disease. N Engl. J Med. 2004;350(10):1005-12.

7. Martinez FJ, Foster G, Curtis JL, Criner G, Weinmann G, Fishman A, et al. Predictors of mortality in patients with emphysema and severe airflow obstruction. Am J Respir Crit Care Med. 2006;173(12):1326-34.

8. Dolan S, Varkey B. Prognostic factors in chronic obstructive pulmonary disease. Curr Opin Pulm Med. 2005;11(2):149-52

9. Jones PW, Agusti AG. Outcomes and markers in assessment of chronic obstructive pulmonary disease. Eur Respir J. 2006;27(4):822-32

10. Skeletal muscle dysfunction in chronic obstructive pulmonary disease. A statement of the American Thoracic Society and European Respiratory Society. Am J Respir Crit Care Med. 1999;159(4 Pt2):S1-S40.

11. O'Donnell DE, Revill SM, Webb KA. Dynamic hyperinflation and exercise intolerance in chronic obstructive pulmonary disease. Am J Respir Crit Care Med. 2001;164(5):770-7.

12. Marin JM, Carrizo SJ, Gascon M, Sanchez A, Gallego B, Celli BR. Inspiratory capacity, dynamic hyperinflation, breathlessness, and exercise performance during the 6 minute walk test in chronic obstructive pulmonary disease. Am J Respir Crit Care Med. 2001;163(6):1395-9.

13. Rodrigues JR, Pereira CAC. Resposta a broncodilatador na espirometria.: que parâmetros e valores são clinicamente relevantes em doenças obstrutivas? J Pneumol. 2001;27(1):35-47.

14. Casanova C, Cote C, de Torres JP, Aguirre-Jaime A, Marin JM, Pinto-Plata V, et al. Inspiratory to total lung capacity ratio predicts mortality in patients with chronic obstructive pulmonary disease. Am J Respir Crit Care Med. 2005;171(6):591-7.

15. Johnson JE. Which exercise test should be used for patients with symptomatic COPD? Chest. 2004;126(3):668-70. 
16. ATS Committee on Proficiency Standards for Clinical Pulmonary Function Laboratories. ATS statement: guidelines for the six-minute walk test. Am J Repir Crit Care Med. 2002;166(1):111-7.

17. Pereira CAC, Sato T, Rodrigues S. Novos valores de referência para espirometria forçada em brasileiros adultos de raça branca. J Bras Pneumol. In press 2007.

18. Neder JA, Andreoni S, Castelo-Filho A, Nery LE. Reference values for lung function tests. Static volumes. Braz J Med Biol Res. 1999;32(6):703-17.

19. Enright PL, Sherril DL. Reference equations for the six minute walk in healthy adults. Am J Respir Crit Care Med. 1998;158(5 Pt 1):1384-7.

20. Pinto-Plata VM, Cote C, Cabral H, Taylor J, Celli BR. The 6min walk distance: change over time and value as a predictor of survival in severe COPD. Eur Respir J. 2004;23(1):28-33.

21. van Stel HF, Bogaard JM, Rijssenbeek-Nouwens LH, Colland VT. Multivariable assessment of the 6-min walking test in patients with chronic obstructive pulmonary disease. Am J Respir Crit Care Med. 2001;163(7):1567-71.

22. Gosselink R, Troosters T, Decramer M. Peripheral muscle weakness contributes to exercise limitation in COPD. Am J Respir Crit Care Med. 1996;153(3):976-80

23. Wijkstra PJ, TenVergert EM, van der Mark TW, Postma DS, Van Altena R, Kraan J, et al. Relation of lung function, maximal inspiratory pressure, dyspnea, and quality of life with exercise capacity in patients with chronic obstructive pulmonary disease. Thorax. 1994;49(5):468-72.

24. Dourado VZ, Antunes LC, Tanni SE, de Paiva SA, Padovani CR, Godoy 1. Relationship of upper-limb and thoracic muscle strength to 6-min walk distance in COPD patients. Chest. 2006;129(3):551-7.

25. Pitta F, Troosters T, Probst VS, Spruit MA, Decramer M, Gosselink R. Physical activity and hospitalization for exacerbation of COPD. Chest. 2006;129(3):536-44.

26. Groenewegen KH, Schols AM, Wouters EF. Mortality and mortality-related factors after hospitalization for acute exacerbation of COPD. Chest. 2003;124(2):459-67.

27. Sociedade Brasileira de Pneumologia e Tisiologia. Diretrizes para Testes de Função Pulmonar. J Pneumol. 2002;28:1-221.

28. O’Donnell DE. Hyperinflation, dyspnea, and exercise intolerance in chronic obstructive pulmonary disease. Proc Am Thorac Soc. 2006;3(2):180-4.

29. Grazzini M, Stendardi L, Gigliotti F, Scano G. Pathophysiology of exercise dyspnea in healthy subjects and in patients with chronic obstructive pulmonary disease (COPD). Respir Med. 2005;99(11):1403-12.

30. Calverley PM. Dynamic hyperinflation: is it worth measuring? Proc Am Thorac Soc. 2006;3(3):239-44. 